\title{
Chemically, Spatially, and Temporally Resolved 2D Mapping Study for the Role of Grain Interiors and Grain Boundaries of Organic-Inorganic Lead Halide Perovskites
}

Gon Namkoong ${ }^{* 1}$, Hyeon Jun Jeong ${ }^{2,3}$, Abdullah Mamun ${ }^{1}$, Hyeryung Byun ${ }^{2,3}$, Derek Demuth ${ }^{1}$, and Mun Seok Jeong ${ }^{* 2,3}$

${ }^{1}$ Department of Electrical and Computer Engineering, Old Dominion University, Applied Research Center, 12050 Jefferson Avenue, Newport News, VA 23606, USA.

${ }^{2}$ Department of Energy Science, Sungkyunkwan University, Suwon 16419, Korea

${ }^{3}$ Center for Integrated Nanostructure Physics, Institute for Basic Science, Sungkyunkwan

University, Suwon 16419, Korea

gnamkoon@odu.edu

mjeong@skku.edu

*Corresponding author:

\begin{abstract}
Grain interiors (GIs) and grain boundaries (GBs) of perovskites have been investigated using chemically, spatially, and temporally resolved measurements. Two dimensional (2D) chemical mapping measurements revealed the GBs consisted of the non-stoichiometric $\mathrm{PbI}_{\mathrm{x}}$ or $\mathrm{CH}_{3} \mathrm{NH}_{3} \mathrm{PbI}_{\mathrm{x}}$, that were characterized by an absence of chloride, an enriched oxygen concentration, and a high density of iodide vacancies. In addition, steady-state 2D photoluminescence showed the bandgap broadening at the GBs while 2D lifetime mapping measurement suggested that the GBs indeed contained deep defect centers. However, it is found that defective GBs in perovskite materials do not act as high recombination sites for photogenerated charge carriers due to the bandgap broadening of non-stoichiometric $\mathrm{PbI}_{\mathrm{x}}$ or
\end{abstract}


$\mathrm{CH}_{3} \mathrm{NH}_{3} \mathrm{PbI}_{\mathrm{x}}$ perovskites at the $\mathrm{GB}$ that forms the potential barriers for photo-generated charge carriers toward the GBs. As a consequence, the photo-generated charge carriers adjacent to the GBs will be easily repelled by the GBs, resulting in a greater reduction of the recombination of photogenerated charge carriers. This is one possible reason for the high performance of $\mathrm{CH}_{3} \mathrm{HN}_{3} \mathrm{PbI}_{3-\mathrm{x}} \mathrm{Cl}_{\mathrm{x}}$ based solar cells.

Keywords: perovskites; grain boundaries; PL mapping; lifetime mapping; non-radiative recombination

\section{Introduction}

Halide perovskites have gained tremendous research interest as a new category of semiconductor materials that will revolutionize photovoltaic technologies. During the last few years, significant improvements in the performance of halide perovskite solar cells have been made, thanks to a new understanding of the materials and new device architecture designs [1-7]. Particularly, spurred world-wide research efforts have revealed the exceptional optical and electrical properties such as high electron and hole mobility, strong defect tolerance, large absorption characteristics resulting from s-p antibonding coupling and the long electron-hole diffusion lengths exceeding $1 \mu \mathrm{m}$ [1-7]. All of these unique characteristics of perovskites led to unprecedented energy conversion efficiency of perovskite solar cells. However, many problems remain to be solved to unravel the underlying mechanisms that will allow for improved photovoltaic performances. The outstanding challenges to be urgently addressed include, but are not limited to, long term stability issues, light soaking, hysteresis, charge carrier dynamics, and 
$\mathrm{Pb}$-free light absorber of perovskites [8-11]. In addition, there's been intensive interest in understanding the nature of grain boundaries $(\mathrm{GBs})$ of perovskites in the solar cell community [12-19]. Since the perovskites are prone to defect formation, due to the low thermal stability of the materials, it is expected that the chemical disorders occur, particularly at the GBs. According to theoretical studies, the GBs are characterized by defects that have very shallow intrinsic levels, i.e. the GBs are completely benign [12]. On the contrary, Agiorgousis et al., also conducted a theoretical calculation and found that the deep charge-state transition levels within the bandgap were indeed possible by forming $\mathrm{Pb}$ dimers and I trimmers through very strong covalent bonds [13].

In addition, experimental studies also revealed controversial results. Till now, the GBs of organic-inorganic halide perovskite films were characterized by confocal fluorescence microscopy, conductive atomic force microscope (c-AFM), Kelvin probe force microscopy (KPFM), and electron beam-induced current (EBIC) [14,16-19]. Nanoscale photoluminescence (PL) and lifetime mappings $[14,16]$ unambiguously revealed that the GBs exhibited strong nonradiative relaxation of charge carriers. This result implies that the GBs are defective and not as benign as previously suggested in ref. 12. In contrast, C-AFM measurements indicate a higher short-circuit current was observed at the GBs than at the grain interiors (GIs), indicating the photo-generated charge carriers more effectively separated at the GBs [17]. Yun et al., also conducted KPFM measurements that revealed greatly enhanced charge separation and collection at the GBs, indicating the benign characteristics of the GBs [17]. In short, the confocal microscope optical measurements $[14,16]$ concluded that the GBs can be detrimental whereas the benign characteristics were unveiled by other measurements $[17,18]$. This begs the question of 
why defective GBs in perovskite materials do not act as high recombination sites for photogenerated charge carriers but enables perovskites solar cells to achieve their high efficiencies. To address this question, we need to clearly understand the nature of the GBs for perovskite solar cells by contrasting them with the GIs. Hence, this study is focused on probing defects at the GIs and GBs, on characterizing the discrete roles of the GIs and GBs mostly influenced by morphological, and chemical variations of perovskites through concerted combinations of chemically, spatially, and temporally resolved microscope studies at the nanoscale. To perform this investigation, we used an energy dispersive X-ray spectroscope (EDS), two-dimensional (2D) photoluminescence (PL) and lifetime to chemically, spatially, and temporally resolve the chemical variations, disorder, and PL decay dynamics across the GBs at the nanoscale.

\section{Experimental details}

Perovskite $\left(\mathrm{PbCH}_{3} \mathrm{NH}_{3} \mathrm{I}_{3-\mathrm{x}} \mathrm{Cl}_{\mathrm{x}}\right)$ films were prepared using hot casting technique. Perovskite $\left(\mathrm{CH}_{3} \mathrm{NH}_{3} \mathrm{I}_{3-\mathrm{x}} \mathrm{Cl}_{\mathrm{x}}\right)$ solution was prepared by dissolving equimolar ratios of lead iodide $\left(\mathrm{PbI}_{2}\right.$, Sigma-Aldrich, 99\%) and methylamine hydrochloride (MACl, Sigma-Aldrich) in N, Ndimethylformamide (DMF, Sigma-Aldrich, anhydrous, 99.8\%) in 11\% concentration. The solution was ready for use after heating in a hot plate at $70{ }^{\circ} \mathrm{C}$ for $24 \mathrm{~h}$ with magnetic stirring in a $\mathrm{N}_{2}$ filled glove box. Glass substrates were cleaned with four times sonication (each for 10 minutes) in de-ionized (DI) water, methanol, acetone, and 2-propanol (IPA) respectively. Afterwards, the substrates were dried with nitrogen followed by heating in a hot plate at $120{ }^{\circ} \mathrm{C}$ for 15 minutes to remove the last traces of any solvent. Glass substrates were kept at different temperatures ranging from 100 to $180{ }^{\circ} \mathrm{C}$ while perovskite solution was kept at $70{ }^{0} \mathrm{C}$. The solution was then immediately deposited on the hot substrate by spin coating at $4000 \mathrm{rpm}$ for $15 \mathrm{~s}$ 
so that the substrate temperature is retained. During this process, the temperature was closely monitored using an IR thermal gun. For a comparison, perovskites were prepared using the hotcasting technique at different temperatures [20,21]. In this case, a lower hot-casting temperature $\left(100{ }^{\circ} \mathrm{C}\right)$ resulted in smaller grain size, with typical grain size of $1 \sim 2 \mu \mathrm{m}$. Hot-casting temperatures higher than $100{ }^{\circ} \mathrm{C}$ produced perovskite samples with larger grains of $\sim$ more than tens of micrometers, which was confirmed by a high-resolution scanning electron microscope (SEM). Grain sizes and morphologies of our samples are consistent with experiments reported in refs $[20,21]$, which also demonstrated the correlation between energy conversion efficiencies of perovskite solar cells and large grains sizes. The PL intensity and peak-position mapping image of submicron-scale were performed using confocal micro-spectroscopy (NTEGRA spectra, NTMDT). In-plane spatial resolution of $\sim 380 \mathrm{~nm}$ was indicated by an objective lens with numerical aperture of 0.7 and a solid-state laser with $405 \mathrm{~nm}$ wavelength for the excitation. The excitation power of $0.2 \mu \mathrm{W} / \mathrm{cm}^{2}$ was used to avoid degradation by laser. The signal collected by the objective lens was detected by a thermoelectrically cooled CCD detector. For analysis of time resolved PL (TRPL), multifunctional confocal microscopy including a time-correlated single photon counting (TCSPC) system was employed (NTEGRA, NT-MDT). For the TRPL excitation source, a $405 \mathrm{~nm}$ pulsed laser with a repetition rate of $50 \mathrm{MHz}$, a pulse width down to $60 \mathrm{ps}$ and excitation power of $0.2 \mu \mathrm{W} / \mathrm{cm}^{2}$ was used. A high-speed PMT detector (PMC-100, Photonic Solutions) was applied for photon counting. A field emission scanning electron microscope (FESEM, JSM7500F, JEOL) was used to investigate the surface morphology of the perovskite solar cells. The elemental compositions of the perovskite films prepared with different hot casting temperatures were carried out using energy dispersive spectroscopy (EDS) mapping in SEM mode. Samples for SEM/EDS were prepared by Pt coating with three times for 
preventing electron charging. In the case of EDS, analysis data excluded Pt information from original EDS data. To analyze the compositions of each component in perovskite films, EDS was calibrated with a series of standard samples including $\mathrm{SiO}_{2}, \mathrm{KCl}, \mathrm{PbF}_{2}, \mathrm{Cu}\left(\mathrm{K}_{\alpha}-8.04 \mathrm{keV}, \mathrm{K}_{\beta}-\right.$ $8.9 \mathrm{keV}$ ) and others. And the measurement uncertainty was estimated to be $\pm 1 \%$ by statistical analysis of a series of observation."

\section{Results and discussion}

To investigate the local chemical compositions of the GIs and GBs for perovskites, an EDS measurement coupled with an SEM was performed. Figure 1 shows the two dimensional (2D) EDS mapping of a higher $\left(180{ }^{\circ} \mathrm{C}\right)$ hot-casting perovskite that highlighted the chemical distributions across the GBs. Remarkably, a higher hot-casting perovskite produced pin-hole free GBs, as shown in SEM image of Figure 1a. Particularly, the chemical components composed of perovskites were carefully examined between the GIs and GBs in order to fully understand the role of GIs and GBs of perovskites. The chlorine signal $(2.621 \mathrm{KeV})$ [22] was clearly distinguished from the $\mathrm{Pb}$ signal $(2.342 \mathrm{KeV})$ as shown in Supplementary Figure S1. The

resultant EDS mapping of $\mathrm{CH}_{3} \mathrm{NH}_{3} \mathrm{PbI}_{3-\mathrm{x}} \mathrm{Cl}_{\mathrm{x}}$ clearly revealed that the contents of lead $(\mathrm{Pb})$ and chloride $(\mathrm{Cl})$ in Figures $1 \mathrm{~b}$ and $1 \mathrm{c}$ were significantly lower at the GBs. Note that the EDS mapping of iodide (I) was not shown, but found to be similar to that of $\mathrm{Pb}$. One of the critical observations is an increase in $\mathrm{O}$ contents when approaching the GB, as shown in Figure 1d. As illustrated in Figure S2 in the Supporting Information, the further comparison of EDS mapping of smaller and larger grain perovskites was shown. Notably, the similar chemical distributions at the GBs for both perovskites were observed, i.e. the chemical components of perovskites were 
concentrated at the GIs, while the GBs were characterized by the higher concentration of oxygen but the deficiency of $\mathrm{Pb}, \mathrm{Cl}$, and $\mathrm{I}$.

Figure 2 shows the quantitative analysis of the variations of the chemical components of perovskites as functions of grain sizes and the positions of perovskites. In this study, the controlled grain sizes of perovskites ranged from a few micrometers to $\sim 60 \mu \mathrm{m}$ in Figure 2a-c. The quantitative atomic percentage of $\mathrm{MAPbI}_{3-\mathrm{x}} \mathrm{Cl}_{\mathrm{x}}$ for representative areas in Figures $2 \mathrm{a}$ and $\mathrm{b}$ was analyzed and listed in Table 1. Interestingly, the density of chemical compositions $(\mathrm{C}, \mathrm{Pb}, \mathrm{Cl}$ and I) of perovskites at the GIs is gradually reduced when the grain sizes of perovskites were reduced, as shown in Figure 2d. Notably, a higher atomic percentage (5\%) of $\mathrm{Cl}$ was found with enlarged grain size $(60 \mu \mathrm{m})$ perovskites, which is contrasted with a negligible amount $(<1 \%)$ of $\mathrm{Cl}$ for smaller grain perovskites. Note that $\mathrm{Cl}$-doping is a common technique for achieving higher open circuit voltage and long diffusion lengths of perovskite solar cells [23-25]. Despite intensive studies to increase the $\mathrm{Cl}$ content, the control of the $\mathrm{Cl}$ content in perovskite is extremely difficult, resulting in the negligible content of $\mathrm{Cl}$ in perovskites [23-25]. As demonstrated, our study suggests the novel way to tune the $\mathrm{Cl}$ content by controlling the grain sizes of perovskite using hot-casting technique. We also found that the grain size of perovskite influenced the $(\mathrm{Cl}+\mathrm{I}) / \mathrm{Pb}$ ratio, as shown in Figure 2e. For instance, for smaller grain $(\sim 4 \mu \mathrm{m})$ perovskites, the GIs yielded a ratio of $\mathrm{Pb}$ to $(\mathrm{Cl}+\mathrm{I})$ close to $1: 2.3$ while the larger grain perovskites led to stoichiometric values of $\sim 1: 3$. It suggests that the enlarged grains of perovskites have the excellent chemical structures. In addition, it is found that the distributions of chemical compositions were quite distinct, especially at the GIs and GBs of perovskites. Figure $2 \mathrm{f}$ illustrates the variations of chemical compositions across the perovskites, showing a gradual 
decrease in the chemical components $(\mathrm{C}, \mathrm{Pb}, \mathrm{I}, \mathrm{Cl})$ of $\mathrm{MAPbI}_{3-\mathrm{x}} \mathrm{Cl}_{\mathrm{x}}$ from the GIs to the GBs while clearly illustrating a drastic increase in oxygen at the GBs.

To quantitatively examine chemical distributions between the GIs and GBs, the representative areas in Figure 2a,b were further analyzed. Particularly, the GIs and GBs showed three distinct discrepancies. First, it is found that the chloride was absent at the GBs for both smaller and larger grain perovskites, which provides a critical insight into the nature of grain boundaries. This is because the presence of $\mathrm{Cl}$ in $\mathrm{MAPbI}_{3}$ plays a critical role in mitigating the formation of defects, but yielding a long diffusion length of charge carriers in perovskites [23-25. The absence of $\mathrm{Cl}$ at the GBs suggests that the GBs are more defective and yield shorter diffusion lengths of charge carriers when compared with the GIs. We thoroughly probed different representative GBs for both perovskites, confirming that the $\mathrm{Cl}$ atoms did not exist at the $\mathrm{GBs}$, but existed only at GIs (see Figure S3 and Table S1). As reported, the reduction mechanism of $\mathrm{Cl}$ in perovskite films was attributed to the release of gaseous $\mathrm{CH}_{3} \mathrm{NH}_{3} \mathrm{Cl}$ through an intermediate phase reaction during thermal annealing [23]. In our case, since the GBs are characterized by disordered structures, it is reasonable to assume that the disruptive chemical structures at the GBs will have weakly bonding chemical configurations and thereby promote the release of gaseous $\mathrm{CH}_{3} \mathrm{NH}_{3} \mathrm{Cl}$ during the hot-casting processes. Additionally, the smaller radii of $\mathrm{Cl}$ compared to those of $\mathrm{Pb}$ and I might also enhance the depletion of $\mathrm{Cl}$ at the $\mathrm{GBs}$, resulting in the absence of $\mathrm{Cl}$ at the GBs. Second, the GBs are characterized by non-stoichiometric $\mathrm{PbI}_{\mathrm{x}}$ and $\mathrm{MAPbI}_{\mathrm{x}}$ for smaller and larger grain perovskites, respectively. Note that the incomplete coverage of perovskites was also observed for smaller grain perovskites. Our EDS measurements indicated $\mathrm{Pb} / \mathrm{I}$ ratios of $\sim 1: 0.7$ and 1:1.2 for $\mathrm{PbI}_{\mathrm{x}}$ and $\mathrm{MAPbI}_{\mathrm{x}}$ at the GBs, largely deviating from a stoichiometric ratio of 1:3, 
indicating a higher density of iodide vacancy. Therefore, the dominant defects at the GBs were the iodide vacancy $\left(\mathrm{V}_{\mathrm{I}}\right)$ for both perovskites. Third, a higher atomic concentration of oxygen at the GBs, compared to the GIs, is found, as shown in Figure S4. Note the level of oxygen at the GBs was similar regardless of the grain sizes of perovskites, while the oxygen contents at the GIs were decreased with increased grain sizes, indicated in Figure S4. An increase in oxygen concentration at the GBs might be related to the iodide vacancy in which the $\mathrm{Pb}$ cations attract oxygen anions to compensate the iodide anion deficiency. Based on our measurements, it is likely that the GBs are characterized by an absence of chloride, a number of iodide vacancies $\left(\mathrm{V}_{\mathrm{I}}\right)$, and a higher concentration of oxygen. In contrast, the GIs showed better structural compositions of perovskite, showing the stoichiometric $\mathrm{MAPbI}_{3-\mathrm{x}} \mathrm{Cl}_{\mathrm{x}}$.

To investigate the local variation of chemical compositions of perovskites on the optical properties of perovskite, steady-state PL and time-resolved lifetime mapping were performed. Figure 3 shows high-resolution PL spatial mapping that correlates topologies to emission spectra of $\mathrm{MAPbI}_{3-\mathrm{x}} \mathrm{Cl}_{\mathrm{x}}$. For the PL and lifetime mapping, poly(methyl methacrylate) (PMMA) was coated on top of the perovskite as the protective layer from moisture and/or atmospheric oxygen. Particularly, it has been proved that PMMA polymer as an optically transparent material exhibited a higher stability under irradiation [6]. Without PMMA coating on perovskite films, it is found the PL intensities and lifetimes gradually varied during 2D mapping due to photodegradation. A laser diode $\left(\lambda_{\mathrm{exc}}=405 \mathrm{~nm}\right)$ was used for optical excitation of the PL mapping, which was scanned over an area of $20 \mu \mathrm{m} \times 20 \mu \mathrm{m}$ of perovskites. The excitation power of 0.2 $\mu \mathrm{W} / \mathrm{cm}^{2}$ was used to avoid the degradation by a laser diode, and spatially integrated emission spectra ranging from 680 to $815 \mathrm{~nm}$ were collected. We note that the PL images are consistent 
with microscopic morphologies which depict a higher contrast at the GBs and suggest significant differences in optoelectronic properties between the GIs and GBs. Overall, the PL intensity of a larger grain perovskite, Figure 3a, is approximately 10 times stronger than that of a smaller grain perovskite, Figure $3 \mathrm{c}$. This suggests that a larger grain perovskite possesses better optical quality. Based on 2D PL mapping, it was found that the GBs for both perovskites were characterized by the strong PL quenching at the GBs, which could be in part attributed to non-radiative trap centers. In addition, it is found that emission spectra were gradually blue-shifted towards the GBs and had an abrupt blue-shift at the GBs, as shown in Figures 3e and 3f. An increase in chemical inhomogeneity towards the GB might be responsible for the non-radiative PL and the blue-shift in PL peaks. Note a blue-shift towards the GBs suggests a gradual broadening of the energy bandgap from GIs towards GBs and an abrupt broadening of the energy bandgap at the GBs.

To get a deeper insight into the recombination kinetics of perovskites, time-resolved PL mapping was investigated with a confocal microscope at a nanoscale resolution of $\sim 380 \mathrm{~nm}$, which allows for spatially resolving recombination kinetics at the GIs and GBs. In this case, the perovskites were excited with a $405 \mathrm{~nm}$ pulsed laser (pulse width down to $60 \mathrm{ps}$, repetition rate $50 \mathrm{MHz}$ ). Figure 4 shows the lifetime mapping of perovskites that are clearly distinguishable between the GIs and the GBs. By quantitatively analyzing the lifetimes across the grains, the average lifetimes for smaller and larger grain perovskites at the GIs, as shown in Figure S5, were estimated about $\sim 1.8$ and $2.5 \mathrm{~ns}$, respectively. Interestingly, a smaller grain perovskite produced a slightly longer lifetime at the GIs by $\sim 1.4$ times than that of a larger grain perovskite. Note that Nie et al. [20], reported a similar observation in which a smaller grain perovskite yielded the 
longer effective carrier lifetime but smaller diffusion constants of minority carriers than that of a larger grain perovskite, emphasizing the disordered and defective natures of a smaller grain perovskite. The carrier lifetime distribution in Figure 4 was further analyzed by contrasting four different transient regions highlighting the hot-spot (donated by red region A), the GI (yellow region B), the area adjacent to the GBs (light blue region C) and the GB (dark blue region D), respectively. Interestingly, hot spots within the grain interiors showed longer lifetimes compared to the surrounding regions for both perovskites as highlighted in Figures $4 \mathrm{c}$ and $4 \mathrm{f}$. Approaching the GBs, i.e. approaching from hot-spot $\mathrm{A}$ to $\mathrm{B}$ to $\mathrm{C}$ and to $\mathrm{D}$ regions, the lifetimes of both perovskites gradually decreased, indicating the non-radiative characteristics towards the GBs. Such variations of lifetimes are closely correlated to the localized chemical variations and the evolution of non-stoichiometric perovskites towards the GBs.

Since the defect with deep levels can only be responsible for non-radiative recombination, the $V_{I}$ - one of dominant defects found from our EDS measurements, must have deep defect levels instead of shallow transition energy level. Recently, Agorgousis et al. [13], suggested that the deep-level of defect state $V_{I}$ can be formed when the Fermi energy is close to the band edge. Otherwise, the formation energy of deep-level $V_{I}$ defects is very high, so that it is unlikely to form deep defects, but leads to the shallow-level of $V_{I}$ defects. However, there will be much possibility that an incorporation of a higher concentration of oxygen at the GBs might shift the position of Fermi energy level close to conduction band. As a consequence, the modification of Fermi level is likely to lower the formation energy of $V_{I}$ defects that have the deep-level of defect states and serve as trap-assisted recombination centers at the GBs. Indeed, a recent experiment demonstrated that the incorporation of $\mathrm{H}_{2} \mathrm{O}$ as an n-type dopant shifted the Fermi 
energy level of the perovskites close to the band edge [26]. Thus, the GBs will contain the deep trap states which might serve as recombination centers. Nevertheless, the GBs might not act as high recombination sites for charge carriers due to the favorably energy bandgap configuration of nonstoichiometric $\mathrm{MAPbI}_{\mathrm{x}}$ or $\mathrm{PbI}_{\mathrm{x}}$ at the GBs, as shown in Figure 5. As observed from PL mapping, $\mathrm{MAPbI}_{\mathrm{x}}$ or $\mathrm{PbI}_{\mathrm{x}}$ at the GBs will have a larger energy bandgap than that of GIs. Given the larger energy bandgap of these materials, they would result in a type I band offset, previously measured by ultraviolet photoemission spectroscopy (UPS) [18]. If such energy bandgap alignment is formed, its beneficial effect would form energy barriers that will prevent charge carriers from the traps states at the GBs, thereby greatly reducing the recombination rates at the GBs. Furthermore, it is expected that such band offset can create the built-in potential, thereby electric field at the GBs that will facilitate the separation of the photogenerated charge carriers. Again, such consequence will lead to the effective reduction of the recombination rates at the GBs. It should be noted that the grain boundary of $\mathrm{Cu}(\mathrm{InGa}) \mathrm{Se}_{2}$ and $\mathrm{Cu}_{2} \mathrm{ZnSnS}_{4}$ solar cell materials has broadening or band bending of the band gap, consequently leading to the suppression of the photogenerated charge carriers at the GBs [27-29]. Therefore, the benign characteristics of the GBs of perovskites observed from many experimental measurements could be originated from a favorable larger energy bandgap alignment at the GBs, even though the $\mathrm{MAPbI}_{\mathrm{x}}$ or $\mathrm{PbI}_{\mathrm{x}}$ formed at the GBs is defective and contains deep trap centers.

\section{Conclusion}

The GIs and GBs of perovskites have been investigated using chemically, spatially, and temporally resolved measurements at the nanoscale. The local variations in steady-state PL and time-resolved lifetime are correlated to chemistries, to nanoscale morphologies, and to 
recombination kinetics. It is found that localized chemical compositions of $\mathrm{MAPbI}_{3-\mathrm{x}} \mathrm{Cl}_{\mathrm{x}}$ perovskites revealed that the GBs were characterized by an absence of chloride, an enriched oxygen concentration, and iodide vacancies, regardless of the gain sizes. In contrast, we found that the content of $\mathrm{Cl}$ at the GIs is strongly dependent upon the grain sizes. Critically, the spatially and temporally resolved PL and lifetime measurements revealed non-radiative characteristics at the GBs, such as very strong PL quenching and relatively shorter lifetimes. The results suggest that the GBs indeed contain deep defect centers that might serves as recombinant centers and be detrimental to the perovskite solar cells. However, the benign characteristics of GBs of perovskites can be originated from the bandgap broadening of non-stoichiometric $\mathrm{MAPbI}_{\mathrm{x}}$ or $\mathrm{PbI}_{\mathrm{x}}$ perovskites at the GB that will form the potential barriers for photo-generated charge carriers toward the GBs. As a consequence, the photo-generated charge carriers adjacent to the GBs will be easily repelled by the GBs, resulting in a greater reduction of the recombination of charge carriers. This is one possible reason for the high performance of $\mathrm{MAPbI}_{3-\mathrm{x}} \mathrm{Cl}_{\mathrm{x}}$ based solar cells.

\section{ACKNOWLEDGMENT}

This work is partially supported by National Science Foundation (NSF) awards \# 1428298, \#1244707 and by Project Code (IBS-R011-D1).

\section{REFERENCES}

[1] M. M. Lee, J. Teuscher, T. Miyasaka, T. N. Murakami and H. J. Snaith, Efficient hybrid Solar Cells Based on Meso-Superstructured Organometal Halide Perovskites, Science 338 (2012) 643-647. 
[2] J. Burschka, N. Pellet, S.-J. Moon, R. Humphry-Baker, P. Gao, M. K. Nazeeruddin and M. Gr"atzel, Sequential deposition as a route to high-performance perovskite-sensitized solar cells, Nature 499 (2013) 16-319.

[3] H. Zhou, Q. Chen, G. Li, S. Luo, T.-b. Song, H.-S. Duan, Z. Hong, J. You, Y. Liu and Y. Yang, Interface engineering of highly efficient perovskite solar cells, Science 345 (2014) $542-546$.

[4] N. J. Jeon, H.G. Lee, Y.C. Kim, J. Seo, J.H. Noh, J. Lee,and S. I. Seok, o-Methoxy Substituents in Spiro-OMeTAD for Efficient Inorganic-Organic Hybrid Perovskite Solar Cells, J. Am. Chem. Soc. 136 (2014) 7837- 7840.

[5] H.S. Kim, C.R. Lee, J.H. Im, K.B. Lee,T. Moehl, A. Marchioro, S.J. Moon, R. HumphryBaker, J.H. Yum, J.E. Moser, M. Grätzel, N.G. Park, Lead Iodide Perovskite Sensitized All-Solid-State Submicron Thin Film Mesoscopic Solar Cell with Efficiency Exceeding 9\%, Sci. Rep. 2 (2012) 591.

[6] S. D. Stranks, G. E. Eperon, G. Grancini, C. Menelaou, M. J.P. Alcocer, T. Leijtens, L. M. Herz, A. Petrozza, and H. J. Snaith, Electron-hole diffusion lengths exceeding 1 micrometer in an organometal trihalide perovskite absorber, Science 342 (2013) 341-344.

[7] N.G. Park, Perovskite solar cells: an emerging photovoltaic technology, Mater. Today 18 (2015) 65-72.

[8] H.S. Jung, N.G. Park, Perovskite Solar Cells: From Materials to Devices, Small 11 (2015) $10-25$.

[9] Q. Chen, N.D. Marco, Y. Yang, T.B. Song, C.C. Chen, H. Zhao, Z. Hong, H. Zhou, Y. Yang, Under the spotlight: The organic - inorganic hybrid halide perovskite for optoelectronic applications, Nano Today 10 (2015) 355-396 . 
[10] W.J. Yin, J.H. Yang, J. Kang, Y. Yan, S.H. Wei, Halide perovskite materials for solar cells: a theoretical review, J. Mater. Chem. A 3 (2015) 8926-8942.

[11] Y. Han, S. Meyer, Y. Dkhissi, K. Weber, J.M. Pringle, U. Bach, L. Spicci, Y.B. Cheng, Degradation observations of encapsulated planar $\mathrm{CH}_{3} \mathrm{NH}_{3} \mathrm{PbI}_{3}$ perovskite solar cells at high temperatures and humidity, J. Mater. Chem. A 3 (2015) 8139-8147.

[12] W.J. Yin, T. Shi, Y. Yan, Unique properties of Halide Perovskites as possible origins of the superior solar cell performance, Adv. Mater. 26 (2014) 4653-4658.

[13] M. L. Agiorgousis Y.-Y. Sun, H. Zeng, and S. Zhang, Strong Covalency-Induced Recombination Centers in Perovskite Solar Cell Material $\mathrm{CH}_{3} \mathrm{NH}_{3} \mathrm{PbI}_{3}$, J. Am. Chem. Soc. 136 (2014) 14570-14575.

[14] D. W. deQuilettes, S. M. Vorpah, S. D. Stranks, H. Nagaoka, G. E. Eperon, M. E. Ziffer, H. J. Snaith, and D. S. Ginger, Impact of microstructure on local carrier lifetime in perovskite solar cells, Science 348 (2015) 683-686.

[15] G.J. A. H. Wetzelaer, M. Scheepers, A. M. Sempere, C. Momblona, J. Ávila, and H. J. Bolink, Trap-Assisted Non-Radiative Recombination in Organic-Inorganic Perovskite Solar Cells, Adv. Mater 27 (2015) 1837-1841.

[16] M. Vrućinić, C. Matthiesen, A. Sadhanala, G. Divitini, S. Cacovich, S.E. Dutton, C. Ducati, M. Atatüre, H. Snaith, R.H. Friend, H. Sirringhaus, F. Deschler, Local Versus Long-Range Diffusion Effects of Photoexcited States on Radiative Recombination in Organic-Inorganic Lead Halide Perovskites, Adv. Sci. 2 (2015) 1500136.

[17] J.S. Yun, A.H. Baillie, S. Huang, S.H. Woo, Y. Heo, J. Seidel, F. Huang, Y.B. Cheng, M.A. Green, Benefit of Grain Boundaries in Organic-Inorganic Halide Planar Perovskite Solar Cells, J. Phys. Chem. Lett. 6 (2015) 875-880. 
[18] Q. Chen, H. Zhou, T.B. Song, S. Luo, Z. Hong, H.S. Duan, L. Dou, Y. Liu, Y. Yang, Controllable Self-Induced Passivation of Hybrid Lead Iodide Perovskites toward High Performance Solar Cells, Nano Lett. 14 (2014) 4158-4163.

[19] E. Edri, S. Kirmayer, S. Mukhopadhyay, K. Gartsman, G. Hodes, D. Cahen, Elucidating the charge carrier separation and working mechanism of $\mathrm{CH}_{3} \mathrm{NH}_{3} \mathrm{PbI}_{3-x} \mathrm{Cl}_{x}$ perovskite solar cells, Nat. Commun. 5 (2014) 3461.

[20] W. Nie, H. Tsai, R. Asadpour, J.C. Blancon, A.J. Neukirch, G. Gupta, J.J. Crochet, M. Chhowalla, S. Tretiak, M.A. Alam, H.L.Wang, A.D. Mohite, High-efficiency solutionprocessed perovskite solar cells with millimeter-scale grains, Science 347 (2015) 522-525.

[21] Y.C. Zheng, S. Yang, X. Chen, Y. Chen, Y. Hou, H.G. Yang, Thermal-Induced VolmerWeber Growth Behavior for Planar Heterojunction Perovskites Solar Cells, Chem. Mater. 27 (2015) 5116-5121.

[22] E.L. Unger, A.R. Bowring, C.J. Tassone, V.L. Pool, A. Gold-Parker, R. Cheacharoen, K.H. Stone, E.T. Hoke, M.F. Toney, M.D. McGehee, Chloride in Lead Chloride-Derived Organo-Metal Halides for Perovskite-Absorber Solar Cells, Chem. Mater. 26 (2014) 71587165 .

[23] F. Xie, W. Li, J. Chen, N. Zhao, The Role of Chlorine in the Formation Process of $\mathrm{CH}_{3} \mathrm{NH}_{3} \mathrm{PbI}_{3-\mathrm{x}} \mathrm{Cl}_{\mathrm{x}}$ Perovskite, Adv. Funct. Mater. 24 (2014) 7102-7108.

[24] S. Colella, E. Mosconi, P. Fedeli, A. Listorti, F. Gazza, F. Orlandi, P. Ferro, T. Besagni, A. Rizzo, G. Calestani, G. Gigli, Angelis, F. D.; Mosca, R. ; MAPbI ${ }_{3-\mathrm{x}} \mathrm{Cl}_{x}$ Mixed Halide Perovskite for Hybrid Solar Cells: The Role of Chloride as Dopant on the Transport and Structural Properties, Chem. Mater. 25 (2013) 4613-4618. 
[25] T.W. Ng, C.Y. Chan, M.F. Lo, Z.O. Guan, C.S. Lee, Formation chemistry of perovskites with mixed iodide/chloride content and the implications on charge transport properties, J. Mater. Chem. A 3 (2015) 9081-9085.

[26] Y. Li, X. Xu, C. Wang, C. Wang, F. Xie, Y. Gao, Degradation by Exposure of Coevaporated $\mathrm{CH}_{3} \mathrm{NH}_{3} \mathrm{PbI}_{3}$ Thin Films, J. Phys. Chem. C 119 (2015) 23996-24002.

[27] M. Takihara, T. Minemoto, Y. Wakisaka, T. Takahashi, An investigation of band profile around the grain boundary of $\mathrm{Cu}(\mathrm{InGa}) \mathrm{Se} 2$ solar cell material by scanning probe microscopy, Prog. Photovolt. 21 (2013) 595-599.

[28] J.B. Li, V. Chawla, B.M. Clemens, Investigating the Role of Grain Boundaries in CZTS and CZTSSe Thin Film Solar Cells with Scanning Probe Microscopy, Adv. Mater. 24 (2012) 720-723.

[29] M. Maiberg, C. Spindler, E. Jarzembowski, R. Scheer, Electrical characterization of $\mathrm{Cu}(\mathrm{In}, \mathrm{Ga}) \mathrm{Se} 2$-solar cells by voltage dependent time-resolved photoluminescence, Thin Solid Films 582 (2015) 379-382.

\section{Figure captions}

Figure 1. (a) SEM images of large grain perovskite and corresponding chemical distributions of (b) lead $(\mathrm{Pb})$, (c) chloride $(\mathrm{Cl})$ and $(\mathrm{d})$ oxygen $(\mathrm{O})$ that were measured by EDS mapping.

Figure 2. SEM images of (a) small $(\sim 4 \mu \mathrm{m})$, (b) medium $(\sim 15 \mu \mathrm{m})$ and (c) large $(\sim 60 \mu \mathrm{m})$ grain perovskites. (d) Atomic percentage of chemical components $(\mathrm{C}, \mathrm{Pb}, \mathrm{Cl}, \mathrm{I}, \mathrm{O})$ and $(\mathrm{e})(\mathrm{Cl}+\mathrm{I})$ to $\mathrm{Pb}$ ratio at the GIs as functions of grain sizes and the positions of perovskites. (f) Atomic percentage of selected areas of I, II, III, and IV across large $(\sim 60 \mu \mathrm{m})$ grains. 
Figure 3. High-resolution photoluminescence (PL) confocal microscopic images and PL intensity spatial mapping that correlate topologies to emission spectra of $\mathrm{CH}_{3} \mathrm{NH}_{3} \mathrm{PbI}_{3-\mathrm{x}} \mathrm{Cl}_{\mathrm{x}}$. (a) and (c) Microscopic image, (b) and (c) corresponding PL intensities of large and small grain perovskites, respectively. (c) and (f) PL mapping showing the blue-shift of emission peaks at the GB for a large grain perovskite shown in (b).

Figure 4. Lifetime mapping of hot-casting perovskites that are clearly distinguishable between the GIs and the GBs. (a) and (d) Time resolved 2D lifetime mapping, (b) and (e) magnified selected lifetime mapping and (c) and (f) corresponding lifetime values as a function of positions for large and small grain perovskites, respectively.

Figure 5. Energy bandgap profile in perovskites outlined, based on the chemical and optical characteristics of EDS, PL and time resolved lifetime measurements. The larger bandgap of $\mathrm{PbI}_{\mathrm{x}}$ or $\mathrm{MAPbI}_{\mathrm{x}}$ at the GBs was formed and had a favorable bandgap alignment, thereby greatly reducing the recombination rate of photogenerated charge carriers at the GBs.

\section{Table caption}

Table 1. Atomic chemical percentage of selected chemical components of small $(\sim 4 \mu \mathrm{m})$ and medium $(\sim 15 \mu \mathrm{m})$ perovskites of Figures $2 \mathrm{a}$ and $\mathrm{b}$. Other chemical elements such as $\mathrm{Na}, \mathrm{Mg}, \mathrm{Al}$, $\mathrm{Si}, \mathrm{S}, \mathrm{Ka}$, and $\mathrm{Ca}$ were detected but not shown here.

\begin{tabular}{ccccc}
\hline & \multicolumn{100}{c}{ hot-casting perovskite } & \multicolumn{1}{c}{$\mathbf{1 8 0}^{\circ} \mathbf{C}$ hot-casting perovskite } \\
\hline & $\begin{array}{c}\text { Grain Interior } \\
(\mathrm{GI})\end{array}$ & $\begin{array}{c}\text { Grain Boundary } \\
(\mathrm{GB})\end{array}$ & $\begin{array}{c}\text { Grain Interior } \\
(\mathrm{GI})\end{array}$ & $\begin{array}{c}\text { Grain Boundary } \\
(\mathrm{GB})\end{array}$ \\
\hline $\mathbf{C}$ & 9.79 & & 19.64 & 12.85 \\
$\mathbf{O}$ & 58.28 & 66.44 & 41.95 & 53.64 \\
$\mathbf{P b}$ & 0.97 & 0.47 & 1.14 & 0.37 \\
$\mathbf{C l}$ & 1.21 & & 0.71 & \\
\hline
\end{tabular}




\begin{tabular}{lllll}
\hline I & 1.01 & 0.34 & 1.91 & 0.43 \\
\hline
\end{tabular}

Table 1.
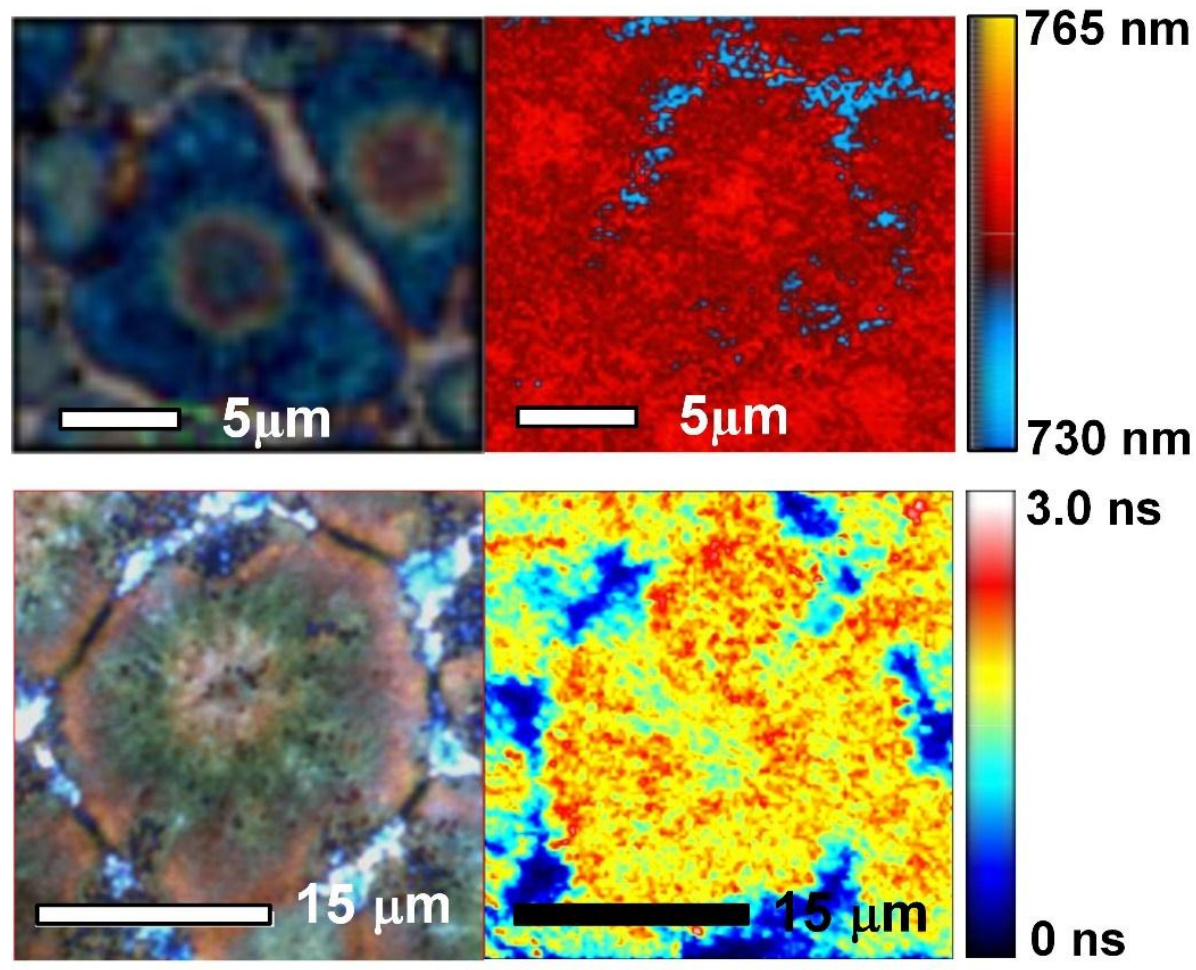

Two-dimensional photoluminescence and time resolved lifetime contrasting the grain interiors and grain boundaries. 
Two-dimensional photoluminescence and time resolved lifetime contrasting the grain interiors and grain boundaries.

\section{Figures}

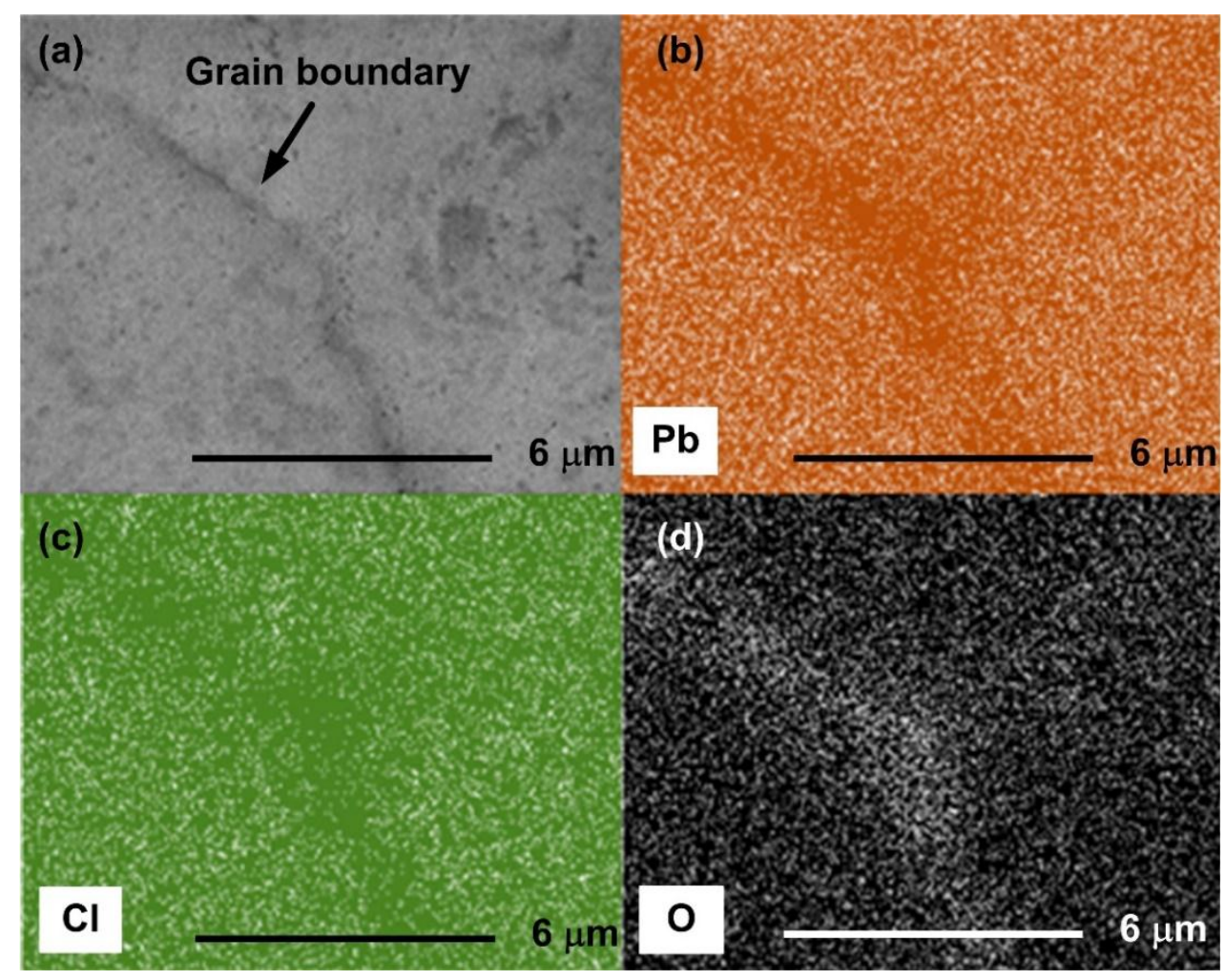

Figure 1. 


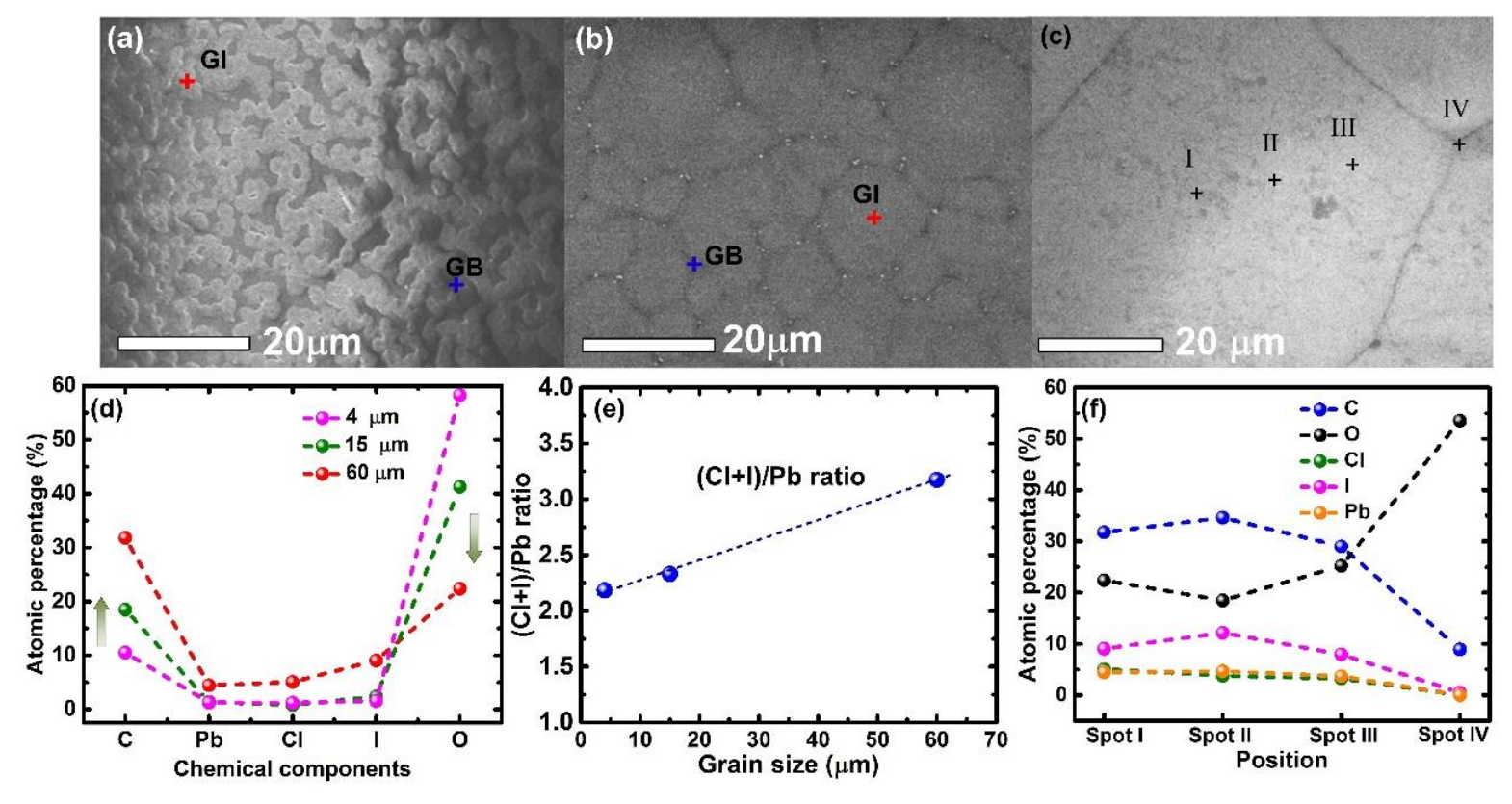

Figure 2 

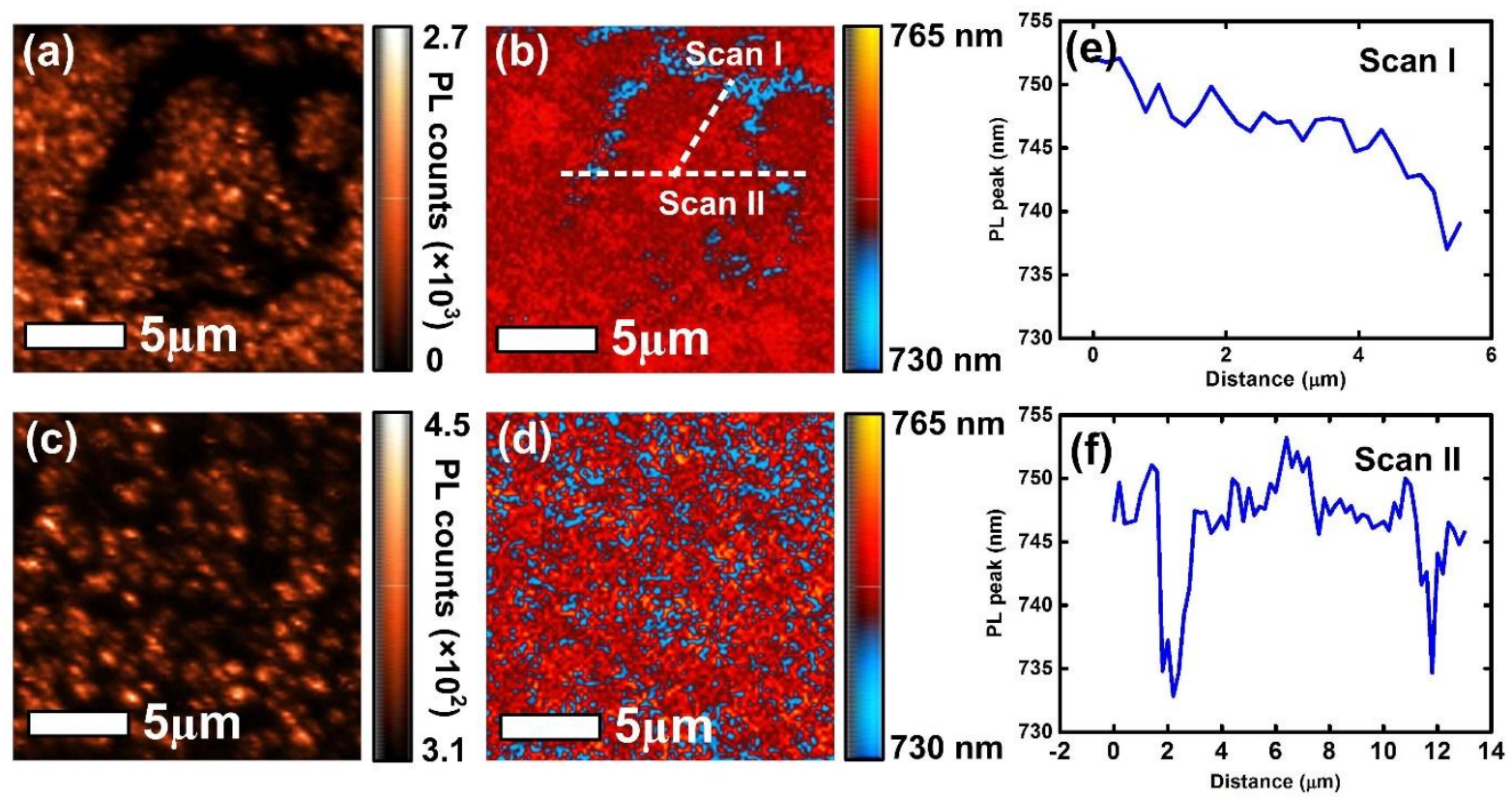

Figure 3 

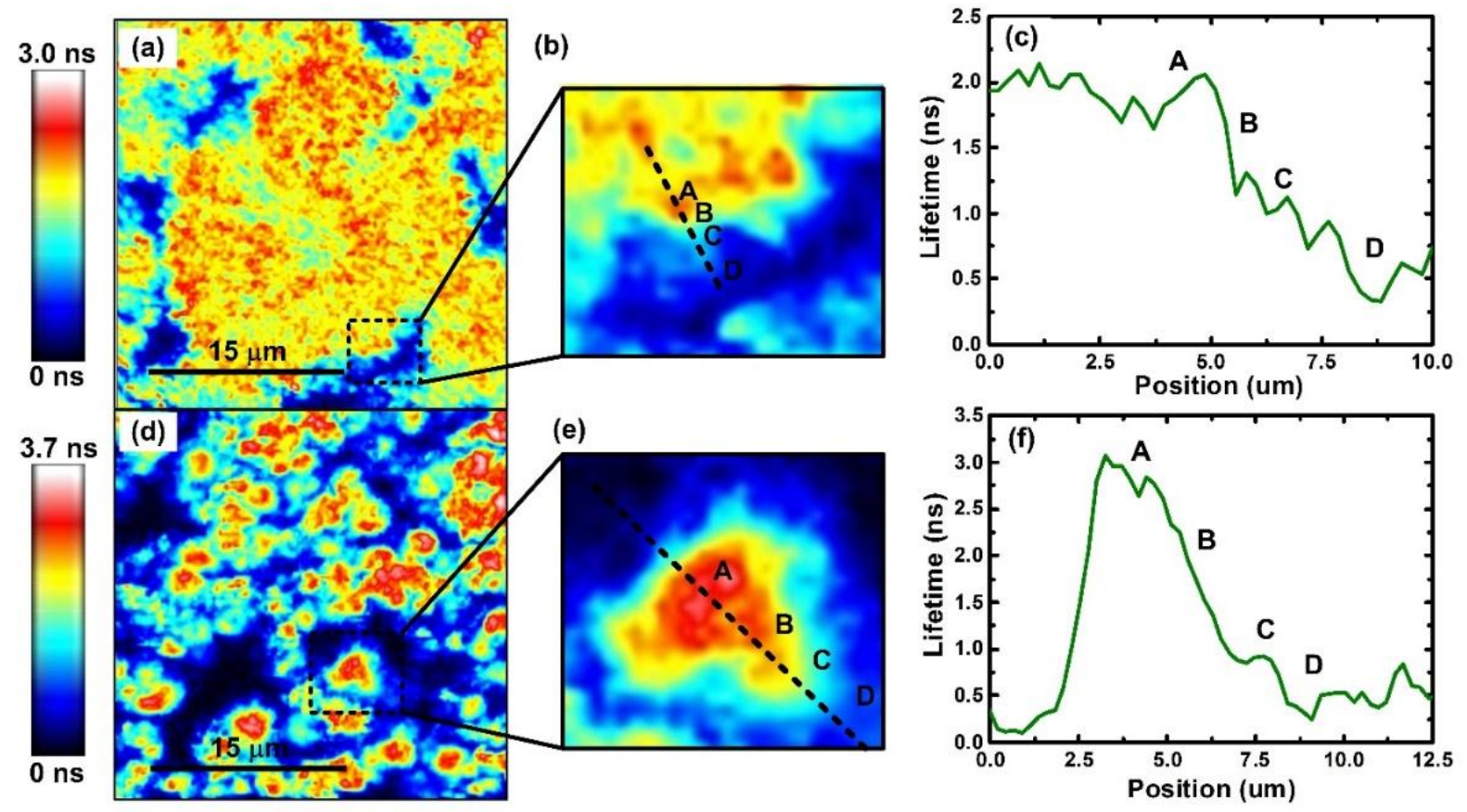

Figure 4 


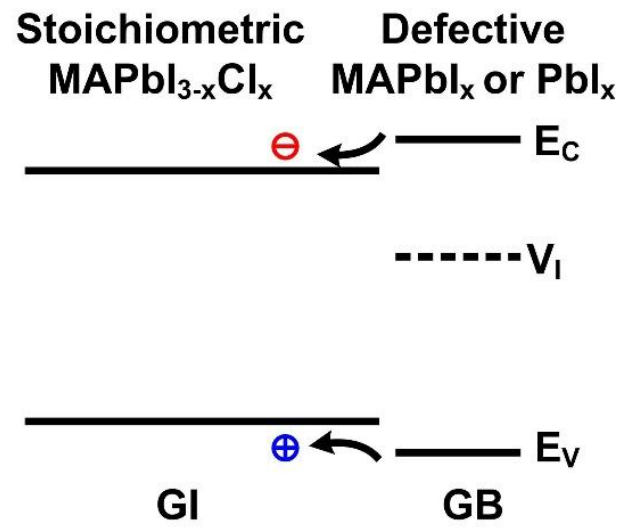

Figure 5 